\title{
Editorial: Chemical Modification of Adsorbents for Enhanced Carbon Capture Performance
}

\author{
Georgios N. Karanikolos ${ }^{1,2,3 *}$, George Em. Romanos ${ }^{4}$ and Lourdes F. Vega ${ }^{1,2,3}$ \\ ${ }^{1}$ Department of Chemical Engineering, Khalifa University, Abu Dhabi, United Arab Emirates, ${ }^{2}$ Research and Innovation Center on \\ $\mathrm{CO}_{2}$ and $\mathrm{H}_{2}(\mathrm{R} / \mathrm{CH})$, Khalifa University, Abu Dhabi, United Arab Emirates, ${ }^{3}$ Center for Catalysis and Separations (CeCaS), Khalifa \\ University, Abu Dhabi, United Arab Emirates, ${ }^{4}$ Institute of Nanoscience and Nanotechnology (INN), Demokritos National Research \\ Center, Athens, Greece
}

Keywords: carbon capture, adsorption, functionalization, $\mathrm{CO}_{2}$, carbon dioxide

Editorial on the Research Topic

Chemical Modification of Adsorbents for Enhanced Carbon Capture Performance

Efficiently capturing carbon dioxide from diverse sources and under different conditions of temperature, pressure and composition is vital in mitigating the impact of continuously increasing $\mathrm{CO}_{2}$ levels into the atmosphere (Alivisatos and Buchanan, 2010; Rangaraj et al.). Of the various techniques, adsorption presents particular prominence mainly due to low energy consumption, cyclability, fast kinetics, versatility of potential adsorbent materials, range of operating conditions, and the fact that it is environmentally-friendly (Karousos et al., 2017; Bahamon et al., 2020; Varghese et al., 2020). In this collection, a variety of adsorbent families among the most promising ones for carbon capture were studied and optimized via various functionalization strategies, i.e., metal-organic frameworks (MOFs), zeolites, porous carbons, Deep Eutectic Solvent (DES)/carbon nanotube (CNT) systems, and graphene oxide adsorbents, as summarized below.

The contribution by Kamran et al. focused on heteroatom-rich porous carbon adsorbents derived from an inexpensive, non-toxic, and easily available polyacrylonitrile (PAN) precursor. The adsorption performance of the resulting activated carbons was optimized by parametrically varying the types of activators used at different temperatures. Namely, $\mathrm{NaOH}, \mathrm{KOH}, \mathrm{K}_{2} \mathrm{CO}_{3}$, and $\mathrm{KNO}_{3}$ were employed and the effect on textural properties and $\mathrm{CO}_{2}$ adsorption performance was evaluated. For the optimum adsorbents, high surface areas were achieved $\left(>3,000 \mathrm{~m}^{2} / \mathrm{g}\right)$ combined with high pore volumes, features that resulted in enhanced $\mathrm{CO}_{2}$ capacities of up to $163 \mathrm{mg} / \mathrm{g}(\mathrm{NaOH}$ activated) at ambient conditions, coupled to high $\mathrm{CO}_{2} / \mathrm{N}_{2}$ selectivity. In addition, the heat of adsorption of the developed adsorbents was relatively low $(<40 \mathrm{~kJ} / \mathrm{mol})$ indicating a physisorption mechanism, which can ensure a small amount of energy required for regeneration, and the possibility of a PSA-based capture process. Indeed, efficient cyclic operation of the developed porous carbons was also demonstrated in the paper.

Thomou et al.performed silylation of organically modified graphene oxide using various silica precursors (APTEOS, BTB, and TEOS) aiming at creating high-yield silica networks as pillars between the organo-modified graphene oxide (GO) layers. Thermal decomposition of the organic moieties endowed the pillared GO with enhanced porosity and resulted in robust 3D structures. XPS and FTIR spectroscopy evidenced reduction of GO to graphene after calcination of the heterostructures and confirmed the presence of silica. The resulting porous materials had a sponge-like structure, with a BET surface area of $550 \mathrm{~m}^{2} / \mathrm{g}$ in the case of G-BTB, and exhibited a $\mathrm{CO}_{2}$ adsorption capacity of up to $3.5 \mathrm{mmol} / \mathrm{g}$ at 5 bar and $0^{\circ} \mathrm{C}$, paving the way

Front Chem 9:657669.

doi: 10.3389/fchem.2021.657669 
for further studies on this type of approach to generate novel porous heterostructured graphene-based adsorbents.

Deep eutectic solvents were employed in the work by Zaib et al. to achieve functional dispersions of CNTs as to promote disaggregation and enhance the carbon dioxide capture performance. Using single walled carbon nanotubes (SWNTs) and Choline Chloride-Glycerol (ChCl-Gly) as DES, the authors explored the effect of critical parameters on the final dispersions, $i$. e. DES concentration in water, sonication energy upon dispersion formation, and concentration of the SWNTs. The impact of the dispersion factors was quantified by determining the average aggregate diameter and the polydispersity index (PDI) of the SWNTs in the aqueous DES systems. Equilibrium thermodynamics and quantum chemistry modeling was also applied, experimentally verified, and statistically validated to map the impact of these factors and to obtain optimized dispersions. Indicatively, optimized dispersions were characterized by small $(<100 \mathrm{~nm})$ and uniform $(<0.1$ PDI $)$ SWNT aggregates obtained at relatively low sonication energy. Such approach is a significant step forward toward development of hybrid (slurry) adsorption/absorption systems, taking advantage of multiple sorption mechanisms for efficient $\mathrm{CO}_{2}$ capture.

Zeolite-based adsorbents and in particular aluminophosphates $\left(\mathrm{AlPO}_{4}-5\right)$ were explored in the work by Papageorgiou et al. Parametric optimization of these adsorbents was achieved by studying the effect of ion substitution ( $\mathrm{Fe}, \mathrm{Mg}, \mathrm{Co}$., and $\mathrm{Si}$ ) into the AFI framework, employing different activation methods (calcination and pyrolysis), and manipulating the reaction mixture dilution rate and the resulting crystal morphology. Upon activation by pyrolysis in inert atmosphere, low temperature treatment $\left(240^{\circ} \mathrm{C}\right)$ enhanced $\mathrm{CO}_{2}$ interaction with the surface at low pressures (up to 1 bar) due to the existence of remnant carbon species in the pores from the partial decomposition of the structure directing agent (SDA). Among the ion-substituted analogues, FeAPO-5 was found to exhibit the highest $\mathrm{CO}_{2}$ capacity at all tested pressures (up to 4 bar). Optimization of the synthesized FeAPO-5 adsorbents led to the material that contained the minimum portion of extra-framework or clustered iron and the highest mesoporosity $\left(\mathrm{Fe} / \mathrm{Al}_{2} \mathrm{O}_{3}\right.$ molar ratio of 5). Furthermore, low

\section{REFERENCES}

Alivisatos, P., and Buchanan, M. (2010). Basic research needs for carbon capture: beyond 2020. Available at: https://www.osti.gov/servlets/purl/ 1291240 (Accessed January 23, 2021).

Bahamon, D., Alkhatib, I. I. I., Alkhatib, N., Builes, S., Sinnokrot, M., and Vega, L. F. (2020). A comparative assessment of emerging solvents and adsorbents for mitigating $\mathrm{CO}_{2}$ emissions from the industrial sector by using molecular modeling tools. Front. Energy Res. 8. doi:10.3389/fenrg.2020. 00165

Karousos, D. S., Labropoulos, A. I., Sapalidis, A., Kanellopoulos, N. K., Iliev, B., Schubert, T. J. S., et al. (2017). Nanoporous ceramic supported ionic liquid membranes for $\mathrm{CO}_{2}$ and $\mathrm{SO}_{2}$ removal from flue gas. Chem. Eng. J. 313, 777-790. doi:10.1016/j.cej.2016.11.005 water content in the synthesis gel led to the formation of spherical agglomerates of small 2D-like crystallites that exhibited higher adsorption capacity compared to columnar-like crystals produced by employing more diluted mixtures. Fast $\mathrm{CO}_{2}$ adsorption kinetics were also obtained from the optimum materials that followed a pseudo-first-order model. The isosteric head of adsorption was low $(<25 \mathrm{~kJ} / \mathrm{mol})$ rendering these materials promising for PSA capture processes.

Amine functionalization of MOFs was explored in the work by Bahamon et al. by means of Grand Canonical Monte Carlo (GCMC) molecular simulations. Different types of aminefunctionalized MOFs were analyzed focusing on optimization for post-combustion $\mathrm{CO}_{2}$ capture. Specifically, six amine models of different chain lengths and degree of substitution grafted to the unsaturated metal sites of the $\mathrm{M}_{2}$ (dobdc) MOF (MOF-74) and its expanded version, $\mathrm{M}_{2}(\mathrm{dobpdc})$, were evaluated in terms of adsorption isotherms, selectivity, cyclic working capacity and regenerability, and compared to experimental data. Among the combinations explored, mmen-Mg/DOBPDC and mda-Zn/ DOBPDC showed high cyclic working capacities upon temperature swing adsorption (TSA). The -mmen functionalized structure in particular showed higher $\mathrm{CO}_{2}$ uptake and better regenerability for the flue gas mixtures and conditions studied. The work also explored the negative effect of steric hindrance and set the associated limits beyond which more amine functional groups grafted on the MOFs and/or full functionalization of the metal centers did not lead to better $\mathrm{CO}_{2}$ separation capability. Such calculations can shed light on how functionalization can enhance gas adsorption via the cooperative chemi-physisorption mechanism of these materials, and how the materials can be tuned to achieve desired adsorption characteristics customized to the conditions of the $\mathrm{CO}_{2}$-containing mixture to be processed.

\section{AUTHOR CONTRIBUTIONS}

GK, GR, and LV contributed in the editing process for this research topic.

Varghese, A. M., Reddy, K. S. K., Singh, S., and Karanikolos, G. N. (2020). Performance enhancement of $\mathrm{CO} 2$ capture adsorbents by UV treatment: the case of self-supported graphene oxide foam. Chem. Eng. J. 386, 124022. doi:10. 1016/j.cej.2020.124022

Conflict of Interest: The authors declare that the research was conducted in the absence of any commercial or financial relationships that could be construed as a potential conflict of interest.

Copyright (c) 2021 Karanikolos, Romanos and Vega. This is an open-access article distributed under the terms of the Creative Commons Attribution License (CC BY). The use, distribution or reproduction in other forums is permitted, provided the original author(s) and the copyright owner(s) are credited and that the original publication in this journal is cited, in accordance with accepted academic practice. No use, distribution or reproduction is permitted which does not comply with these terms. 